\title{
Liderazgo, autoconcepto y sexo: analizando en profundidad el liderazgo transformacional
}

María Pasadas Martín, Ana Isabel Peregrina Rivas, Cristina Prieto Nieto, Víctor Moreno Jiménez. Universidad de Granada

Recepción: 12 de mayo de 2015 | Revisión: 17 de junio de 2015 | Aceptación/Publicación: 17 de julio de 2015

Citar: Pasadas, M., Peregrina, A.I., Prieto, C. y Moreno, V. (2015). Liderazgo, autoconcepto y sexo: analizando en pronfundidad el liderazgo transformacional. ReiDoCrea, 4, 112-116.

Resumen: Una parte de la investigación sobre liderazgo se ha centrado en los efectos del liderazgo transformacional (TF) y transaccional (TA) en el autoconcepto del propio líder. Esta investigación se ha focalizado esta cuestión, debido a la importancia que encierra. Si se distingue qué tipo de liderazgo conlleva poseer un autoconcepto más positivo, es decir, una mayor autoestima, podremos concluir que existe una forma de liderar que, al menos en ese aspecto, es más recomendable. Según Sosik y Cameron (2010), el líder transformacional poseerá una personalidad más ascética y una excelencia moral; y por ello, un autoconcepto más positivo. Además, también analizaremos si existe diferencia en cuanto al sexo a la hora de ejercer un tipo de liderazgo u otro; pues se espera que las mujeres tiendan a desarrollar un liderazgo más transformacional (Eagly y col., 1992). Utilizando como muestra a 56 profesores/as de secundaria de Granada procedimos a pasar una serie de instrumentos que miden el tipo de liderazgo y el autoconcepto, con el fin de poner a prueba nuestras hipótesis. Finalmente, éstas fueron contrastadas, pues se encontró una clara tendencia por parte de las mujeres al liderazgo transformacional, además de comprobar que éste conllevaba un mayor autoconcepto personal y social en las personas que lo elegían como forma de liderazgo a ejercer.

Palabras clave: autoconcepto, transaccional, transformacional, sexo.

Leadership, self-concept and sex: a deep analysis of transformational leadership

Abstract: Part of the research about leadership has been focused on the effects that both transformational and transactional leadership have on the leader's self-concept. This study is focused on that question, due to the importance that it has nowadays. Thus, if we can determine which form of leadership is related to a more positive self-concept, we will be able to conclude that it actually exists a way to lead that is more advisable than others, at least in this respect. Sosik and Cameron (2010) claim that transformational leaders have a more ascetic personality and also a moral excellence, so their self-concept will be more positive. Also, we will analyze the difference between men and women when it comes to lead: we expect that women tend to develop a transformational leadership (Eagly et al., 1992). For that aim, it was drawn a sample of 56 secondary school teachers from Granada, who had to complete different tests about their form of leadership and their self-concept. We finally found that, in general, women tend to have a transformational leadership, and this kind of leadership is related to a better self-concept, both social and personal.

Key words: transactional, transformational, self-concept, sex.

\section{Introducción}

Una gran parte de la investigación sobre liderazgo se ha centrado en los efectos del liderazgo transformacional y carismático en la motivación de los seguidores y su rendimiento (Avolio, 1999; Bass, 1985; Bass y Avolio, 1994, 1997; Conger y Kanungo, 1988; Lowe y Gardner, 2000).

Una de las nuevas teorías de liderazgo (Bryman, 1992) ha sido denominada "FullRange Leadership Theory" (FRLT) o, la teoría de liderazgo de rango completo propuesta por Avolio y Bass en 1991. Los constructos que comprende la teoría de liderazgo de rango completo recogen tres tipologías de comportamientos que puede tener un líder: liderazgo transformacional, liderazgo transaccional y liderazgo no transaccional laissez-faire (Antonakis, Avolio y Sivasubramaniam, 2003). Sin embargo, es importante señalar que para este estudio concreto sólo hemos usado las dimensiones transaccional y transformacional, incluyendo el laissez-faire como un 
componente para medir el liderazgo transaccional debido a las características del instrumento utilizado para medir liderazgo (Multifactor Leadership Questionnaire).

En lo referente a las teorías de liderazgo completo, Bass (1985) afirmó que el liderazgo transaccional estaba limitado a intercambios básicos entre el líder y los subordinados únicamente. Bass sugirió que era necesario un desplazamiento de este paradigma para entender cómo los líderes tienen influencia en los seguidores. Influencia que sobrepasa el propio interés de los seguidores, y que busca conseguir el bien superior de sus organizaciones para conseguir en nivel óptimo de rendimiento. Él se refería a este tipo de liderazgo como liderazgo transformacional. En su forma actual, la teoría de liderazgo de rango completo presenta nueve factores de orden individuales englobados en cinco factores de liderazgo transformacional, tres factores de liderazgo transaccional, y un factor de liderazgo no transaccional laissez-faire (Antonakis y col., 2003).

Así, los líderes transformacionales son proactivos, incrementan la consciencia de los seguidores por los intereses colectivos, y ayudan a los seguidores a conseguir metas extraordinarias. Por su parte, el liderazgo transaccional podría definirse como un proceso de intercambio basado en la cumplimentación de las obligaciones contractuales y es típicamente representado como el ajuste de objetivos y la monitorización y control de las respuestas. (Antonakis y col., 2003).

Antes de tratar su relación con el liderazgo, conviene aclarar el término autoconcepto. Purkey (1970) define el autoconcepto como "un sistema complejo y dinámico de creencias que un individuo considera verdaderas respecto a sí mismo teniendo cada creencia un valor correspondiente". Shavelson, Hubner y Stanton (1976) completan la definición indicando que el autoconcepto no es más que las percepciones que una persona mantiene sobre sí misma formadas a través de la interpretación de la propia existencia y el ambiente, siendo influenciadas, de manera especial, por los refuerzos y feedback de los otros significativos así como los propios mecanismos cognitivos tales como las atribuciones (González-Pienda y col., 1997).

El autoconcepto comprende una doble vertiente: la descriptiva o autoimagen (cómo percibo qué soy) y la valorativa o autoestima (cómo valoro mi autoimagen). La autoimagen que cada uno construimos se encuentra formada tanto por feedback respecto a nosotros como individuos como por información derivada de los roles que desempeñamos en nuestra interacción social (González-Pienda y col., 1997).

En este estudio se sugiere que los auténticos líderes transformacionales, que son conscientes de sus comportamientos, tienen mayor probabilidad de desarrollar una autoconstrucción ascética. Jones (1995) describió la personalidad ascética como la predisposición para vivir sacando lo mejor de uno mismo y los otros y un carácter ético que es moldeado y controlado por el poder de una fuerza interna que llama a la excelencia moral reflejada en el líder (Sosik y Cameron, 2010).

Para el presente estudio y como variables más representativas del autoconcepto, se han seleccionado las dimensiones social y personal de la herramienta Tennesse SelfConcept Scale. Según lo explicado anteriormente, se espera que los líderes transformacionales obtengan un mayor puntaje en ambas dimensiones en contraposición con los líderes transaccionales.

En lo referente a la relación entre el sexo y las variables mencionadas, estudios anteriores han examinado el sexo del líder como foco para encontrar las diferencias y 
semejanzas en estilo de liderazgo de hombres y mujeres (Eagly y Carli, 2007; Eagly y Johnson, 1990; Eagly, Makhijani, y Klonsky, 1992).

Un meta-análisis de las diferencias de sexo en estilos de liderazgo transformacional y transaccional entre líderes actuales encontró que las mujeres puntuaban más que los hombres en la mayoría de las dimensiones de liderazgo transformacional (Eagly et al., 1992). Teniendo en cuenta los resultados, el liderazgo transformacional parece estar más relacionado con cómo lideran las mujeres en comparación con los hombres (Douglas C, 2012).

\section{Método}

\section{Participantes}

Se tomó una muestra de 56 participantes, profesores de Educación Secundaria y Bachillerato de Granada, de los cuales 36 eran mujeres y 20 hombres, de media de edad de 45 años.

\section{Instrumentos}

A los participantes se les pasó una versión española del MLQ5x con el fin de analizar su tipo de liderazgo. Este test posee 45 ítems que se responden en base a una escala tipo Likert teniendo en cuenta que cada número hace referencia a la frecuencia con la cual los participantes pueden aplicarse las afirmaciones propuestas $(0=$ casi nunca; 3 = casi siempre). Este cuestionario posee una a de Cronbach de 0'862.

A continuación se les pasó un conjunto de ítems pertenecientes al test Tennesee SelfConcept Scale, concretamente aquellos que hacían referencia al autoconcepto social y personal. Finalmente, el test quedó reducido a 12 ítems cuya respuesta se basa en una escala tipo Likert que hace referencia al grado de veracidad de cada afirmación (1: completamente falsa; 5: completamente verdadera). De este cuestionario, 6 ítems requerían que se les invirtiera la puntuación. El a de Cronbach de este cuestionario es de 0'557.

\section{Procedimiento}

Los cuestionarios fueron pasados en un plazo de dos días en varios centros de enseñanza secundaria y bachillerato de la provincia de Granada, durante las horas libres del profesorado.

En primer lugar, se les pasó una hoja de consentimiento en la que se especificaban los objetivos del estudio, así como la confidencialidad y carácter voluntario de la participación del estudio. Además, se les explicó que ésta sería retirada del cuadernillo para garantizar el anonimato durante el análisis de los datos. A continuación, y adjunto al consentimiento, se pasaron ambos cuestionarios, además de preguntas acerca de su sexo y edad.

\section{Resultados}

Una vez analizada la muestra con las apropiadas herramientas estadísticas podemos observar: (1) una alta correlación positiva $(<0.01)$ entre la dimensión personal del autoconcepto y el liderazgo transformacional; (2) una alta correlación $(<0.05)$ entre la dimensión social del autoconcepto y el liderazgo transformacional (tabla 1). 


\begin{tabular}{|lccc|}
\hline \multicolumn{4}{|c|}{ Tabla 1 } \\
\hline \multicolumn{4}{|c|}{ Correlación entre tipos de liderazgo y autoconcepto } \\
\hline & Autoconcepto social & Autoconcepto personal & Autoconcepto \\
\hline Liderazgo transformacional & $.327^{* *}$ & $.416^{* *}$ & $.441^{* *}$ \\
\hline Liderazgo transaccional & -.203 & -.136 & -.212 \\
\hline$*$ *. La correlación es significativa al nivel 0,05 (bilateral) & \\
\hline
\end{tabular}

Por otra parte se realizó una $\mathrm{T}$ de Student para comparar las diferencias existentes entre hombres y mujeres en cuanto a liderazgo transformacional. Se obtuvo una puntuación media de 3.172 en liderazgo transformacional en mujeres y una media de 2.827 en hombres. La prueba T de Student para muestras independientes indicó que las mujeres presentaban mayor autoconcepto que los hombres ( $t(54)=2.708, p<$ $.05)$.

\section{Discusión}

Una vez analizados los datos podemos afirmar que los líderes transformacionales presentan una mayor puntación en las dimensiones de autoconcepto social y personal, luego, personas que presenten este estilo de liderazgo tenderán a tener unas creencias más positivas de sí mismos y una mayor autoestima. Esto coincide con nuestra hipótesis de que las personas que presentan rasgos transformacionales de liderazgo obtienen mayores puntuaciones en autoconcepto posiblemente causado por el feedback recibido por sus subordinados en el desempeño de su rol social. También coincide con la propuesta de Sosik y Cameron (2010) en la que se propone que el líder transformacional tiene un buen autoconcepto porque posee una personalidad más ascética, es decir, predispuesta a vivir sacando lo mejor de uno mismo y los otros, es más consciente de sus puntos fuertes y muestra un carácter ético caracterizado por una excelencia moral.

No obstante, no hemos podido encontrar correlaciones significativas positivas o negativas entre autoconcepto y liderazgo transaccional, por lo que no es posible realizar una comparación entre los estilos. Sería interesante comprobar en futuros estudios si esto podría indicar que el líder transaccional, al tener menor implicación con los miembros del grupo, no recibe un feedback de buena calidad (positivo o negativo) por lo que no tendría efecto en su autoconcepto. O si por el contrario es el autoconcepto lo que permite a los líderes expresar sus puntos fuertes de forma más o menos eficiente.

Además en nuestro estudio hemos encontrado diferencias significativas entre sexos en el estilo de liderazgo, mostrando las mujeres una mayor predisposición al estilo transaccional que los hombres. Por lo tanto, nuestra segunda hipótesis queda también confirmada, en consonancia con los datos del meta-análisis de Eagly y cols. (1992): las mujeres tienden desempeñar el rol de líder de forma más transformacional que los hombres.

En resumen, las hipótesis planteadas en el estudio se cumplen: los líderes transformacionales tienen mejor autoconcepto. Además, los datos obtenidos en nuestro estudio reafirman la existencia de una relación entre un mejor autoconcepto y el liderazgo transformacional. Lo que es importante, no solo para poder asegurar que existe una relación entre el tipo de liderazgo que se ejerce y nuestro autoconcepto, sino también para saber que, probablemente, modificando el primero hacia un estilo más transformacional podemos cambiar nuestra forma de percibirnos, para así vernos de una manera más positiva. De igual modo, si cambiamos nuestro autoconcepto nuestra forma de liderar también se verá modificada. 


\section{Referencias}

Antonakis, J., Avolio, B. J. y Sivasubramaniam, N. (2003). Context and leadership: an examination of the nine-factor full-range leadership theory using the multifactor leadership questionnaire. The Leadership Quarterly, 14, 261295.

Avolio, B. J. (1999). Full leadership development: building the vital forces inn organizations. Thousand Oaks, CA: Sage Publications.

Avolio, B. J., y Bass, B. M. (1991). The full range leadership development programs: basic and advanced manuals. Binghamton, NY: Bass, Avolio y Associates.

Avolio, B. J., Waldman, D. W., y Yammarino, F. L. (1991). Leading in the 1990's: towards understanding the four l's of transformational leadership. Journal of European Industrial Training, 15(4), 9-16.

Bass, B. M. (1985). Leadership and performance beyond expectations. New York: Free Press.

Bass, B. M., y Avolio, B. J. (Eds.) (1994). Improving organizational effectiveness through transformational leadership. Thousand Oaks, CA: Sage Publications.

Bass, B. M., y Avolio, B. J. (1997). Full range leadership development: manual for the Multifactor Leadership Questionnaire. Palo Alto, CA: Mindgarden.

Bass, B. M. (1985). Leadership and performance beyond expectations. New York: Free Press.

Bass, B. M. (1998). Transformational Leadership: industrial, military, and educational impact. Mahwah, NJ: Erlbaum.

Bryman, A. (1992). Charisma and Leadership in Organizations. London: Sage Publications.

Conger, J. A., y Kanungo, R. N. (Eds.) (1988). Charismatic leadership: the elusive factor in organizational effectiveness. San Francisco: Jossey-Bass.

Douglas, C. (2012). The moderating role of leader and follower sex in dyads on the leadership behavior-leader effectiveness relationship. The Leadership Quarterly, 23, 163-175.

Eagly, A. H., Makhijani, M. G. y Klonsky, B. G. (1992). Gender and the evaluation of leaders: A meta-analysis. Psychological Bulletin, 111, 3-22.

González-Pienda, J. A., Núñez, J.C., Glez-Pumariega, S. y García, M.S. (1997). Autoconcepto, autoestima y aprendizaje escolar. Psicothema, 9, 271-289.

Hunt, J. G. (1999). Transformational/charismatic leadership's transformation of the field: an historical essay. The Leadership Quarterly, 10, 129-144.

Jones, H. B. (1995). The ethical leader: An ascetic construct. Journal of Business Ethics, 14, 867-874.

Lowe, K. B., y Gardner, W. L. (2000). Ten years of The Leadership Quarterly: contributions and challenges for the future. Leadership Quarterly, 11, 459-514.

Purkey, W.W. (1970). Self-concept and school achievement. Englewood Cliffs, NJ: Prentice Hall.

Shavelson, R.J., Hubner, J.J. y Stanton, G.C. (1976). Validation of construct interpretations. Review of Educational Research, 46, 407-441.

Sosik, J.J. y Cameron, J.C. (2010). Character and authentic transformational leadership behavior: expanding the ascetic self toward others. Consulting Psychology Pournal: Practice and Research, 62, 251-269. 